Moreover, for all values of $z$ within a region $T$ which does not cut or touoh the positive half of the real axis we shall have $-\pi<\phi+\pi<\pi$, provided we agree to choose $\phi$ at every point so that $-2 \pi<\phi<0$. It follows, upon introdueing (6), that when the above agreements are made we may always choose $\epsilon$ so small that the improper integral in (7) will converge uniformly for all values of $z$ in $T$. Whence, the same integral and hence also the second member of (7) will have the analytic properties indicated above.*

Thus we reach in summary the theorem stated at the beginning.

It may be observed that in case the function $g(w)$ satisfies the conditions demanded except that it has a finite number of singularities in the region of the $w$ plane lying to the right of the line $w=a-\frac{1}{2}+i y$ the theorem continues true provided we subtract from the second member of (3) the sum of the residues of the function

$$
\frac{\pi g(w)(-z)^{w}}{\sin \pi w}
$$

corresponding to such singularities.

UNIVERSITY OF Michigan, May, 1910.

\title{
EXTENSIONS OF TWO THEOREMS DUE TO CAUCHY
}

BY PROFKSSOR G. A. MILLER.

(Read before the Chicago Section of the American Mathematical Society, April 9, 1910.)

THE last one of the noted series of papers on substitution groups published by Cauchy during 1845-6 in the Paris Comptes Rendus is devoted to a simplification of his earlier proof of an important theorem which may be stated as follows: If the symmetric group $G$ of degree $n$ involves at least one substitution which transforms one of its subgroups $H_{1}$ into a group having only identity in common with the subgroup $H_{2}$, the total number of such substitutions in $G$ is divisible by the product of the orders of $H_{1}$ and $H_{2}$. The proof given by Cauchy is

\footnotetext{
* Cf. Osgood, Encyklopädie, II, p. 21.
} 
very simple and applies equally when $G$ in any group involving $H_{1}$ and $H_{2}$, as has been observed by Jordan * and others.

The object of the present note is to extend this theorem by modifying only very slightly the method of proof employed by Cauchy and to indicate how easily Sylow's theorem may be obtained from this extension. By doing this we hope to give one of the simplest proofs of Sylow's theorem and to exhibit, at the same time, how close Cauchy, Jordan, and others were to this fundamental theorem a number of years before it was announced by Sylow. While the historical setting is a prominent element of the present note, the subject matter appears sufficiently fundamental to justify various forms of presentation and emphasis on slight extensions.

Let $H_{1}=1, s_{2}, s_{3}, \ldots, s_{h_{1}}$ and $H_{2}=1, t_{2}, t_{3}, \ldots, t_{h_{2}}$ be two subgroups of any group $G$ and suppose that $H_{2}$ and $s^{-1} H_{1} s$, $s$ being any operator of $G$, have exactly $\rho$ operators in common. These $\rho$ common operators form a common subgroup of $H_{1}$ and $H_{2}$. In the following rectangular array of $h_{1} h_{2}$ operators

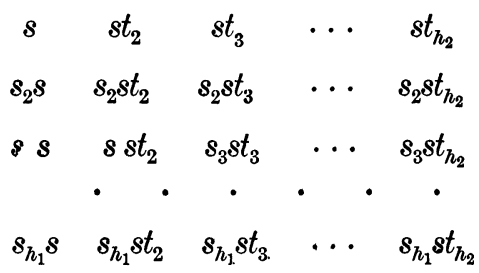

the operators of the same column transform $H_{1}$ into the same group, and each of the operators of this array transforms $H_{1}$ into a group which has exactly $\rho$ operators in common with $H_{2}$. The supposition that two operators of the array are equal implies an equation of the form

$$
s_{\alpha} s t_{\beta}=s_{\gamma} s t_{\delta}, \quad \text { or } \quad s^{-1} \cdot s_{\gamma}^{-1} s_{\alpha} \cdot s=t_{\delta} t_{\beta}^{-1} .
$$

If we suppose $a$ and $\beta$ fixed, $\gamma$ can be chosen in exactly $\rho$ ways so as to satisfy the latter equation. That is, there are exactly $\rho$ operators in the given array which are equal to any given operator of the array. In other words, this array involves exactly $h_{1} h_{2} \div \rho$ distinct operators.

If $s^{\prime}$ is any operator of $G$ which transforms $H_{1}$ into a group having exactly $\rho$ operators in common with $\mathrm{H}_{2}$ but is not in-

*Traité des substitutions, 1870 , p. 26. 
cluded in the given array, we may form another array which can be obtained by replacing $s$ by $s^{\prime}$ in the one given above. This new array must again involve exactly $h_{1} h_{2} / \rho$ distinct operators and none of these can be equal to one of the preceding array since the equation

$$
s_{\alpha} s^{\prime} t_{\beta}=s_{\gamma} s t_{\delta}
$$

implies that $s^{\prime}$ is in the former array. As this process may be repeated until all the operators of $G$ which transform $H_{1}$ into a group having exactly $\rho$ operators in common with $H_{2}$ have been exhausted, we have proved the following theorem :

In any group $G$ the number of distinct operators which transform any subgroup $H_{1}$ into a group having exactly $\rho$ operators in common with a subgroup $H_{2}$ is a multiple of $h_{1} h_{2} / \rho, h_{1}$ and $h_{2}$ being the orders of $H_{1}$ and $H_{2}$ respectively.*

For the special case $\rho=1$ this theorem was proved by Cauchy in the article noted above; since Cauchy used almost the same method as we employed, the present theorem should be regarded as merely a slight extension of the one given by him. Suppose now that $G$ contains a Sylow subgroup $K_{1}$ of order $p^{a}$ and that $K_{2}$ is any other subgroup of $G$, the order of $K_{2}$ being divisible by $p^{\beta}$ but not by $p^{\beta+1}$. We proceed to prove that it follows from the given theorem that $K_{2}$ must involve a Sylow subgroup of order $p^{\beta}$. If $K_{2}$ did not contain a subgroup of order $p^{\beta}$, it would involve a subgroup of order $p^{\beta^{\prime}}, \beta^{\prime}<\beta$, but no subgroup of order $p^{\beta^{\prime}+1}, \beta^{\prime}$ being properly chosen. In this case the number of operators of $G$ which transform $K_{1}$ into a group having no more than $p^{\beta^{\prime}}$ operators in common with $K_{2}$ would be divisible by $p^{\alpha+\beta-\beta^{\prime}}$. In other words, the order of $G$ would be divisible by $p^{\alpha+\beta-\beta^{\prime}}$. As this is contrary to the hypothesis that $K_{1}$ is a Sylow subgroup of $G$, the given theorem implies the corollary :

If a group $G$ involves a Sylow subgroup of order $p^{m}$, each of the subgroups of $G$ whose order is divisible by $p$ contains at least one Sylow subgroup whose order is a power of $p$.

From the preceding paragraph it results that the given theorem implies that if a group $G$ involves at least one Sylow sub-

* This theorem evidently remains true when $H_{1}$ coincides with $H_{2}$ as well as when either one of these subgroups coincides with the entire group $G$. In the latter case it reduces to the theorem that the order of every subgroup divides the order of the group. This special case is sometimes called Lagrange's theorem. 
group for every prime divisor of its order every subgroup of $G$ has the same property. In particular, if the symmetric group of degree $n$ involves Sylow subgroups for every prime which divides its order, then every substitution group of degree $n$ (and hence every group of finite order) must involve at least one Sylow subgroup for every prime which divides its order. It is very easy to prove, as Cauchy observed, that every symmetric group of degree $n$ has the given property, and hence the theorem which was proved above as a slight extension of one due to Cauchy implies that every group of finite order involves at least one Sylow subgroup for every prime divisor of its order. As this is the main element in Sylow's theorem it is clear that Cauchy used a method which required only slight changes to yield an easy proof of the fundamental theorem known as Sylow's theorem. It would evidently be necessary only to prove that every symmetric group whose degree is a power of $p$ involves Sylow subgroups of order $p^{m}$ in order to establish the existence of Sylow subgroups in every group of finite order by means of the theorem proved above.

The preceding remarks may also serve to exhibit additional reasons for regarding Sylow's theorem as merely an extension of Cauchy's fundamental theorem, which established the fact that every group whose order is divisible by the prime $p$ involves operators of order $p$. In fact, if Cauchy had used a general value of $\rho$ instead of $\rho=1$ in the theorem proved above, he would have arrived at Sylow's theorem by the same steps as those which led him to his fundamental theorem. The oversight of this slight increase in generality retarded Sylow's theorem nearly thirty years and made Jordan's Traité des Substitutions much more difficult reading.

\section{EXISTENCE THEOREMS FOR CER'TAIN UNSYM- METRIC KERNELS.}

BY MRS. ANNA J. PELL.

Is this paper is given a brief account of the existence and expansion theorems for certain integral equations with unsymmetric kernels. Full details of the method involved and a discussion of a less general integral equation are contained in an article, "Biorthogonal systems of functions with applica- 